\begin{tabular}{ccc}
\hline International Journal of Engineering \& Technology, $7(2.29)(2018) 1010-1014$ \\
SPC & Website: www.sciencepubco.com/index.php/IJET \\
Research paper & Intional Journal of Engineering \& Technology \\
\hline
\end{tabular}

\title{
Proposed Ethical Work Behavior Model in Boundaries of Organizational Domain
}

\author{
Behjati Saeed $^{1 *}$, Altounjy Rana ${ }^{2}$ \\ ${ }^{1}$ Department Of Management, Roudehen Branch, Islamic Azad University, Roudehen, Iran \\ ${ }^{2}$ Business School, Universiti Kuala Lumpur, Malaysia \\ *Corresponding Author E-Mail: Behjati@Riau.Ac.Ir
}

\begin{abstract}
The organizational code of ethic plays a big role as linkage between the organization's mission, values and principles with the standards of professional conduct. Due to this, the objective of this research is to identify the ethical work behavior and depict a baseline to predict the organizational code of ethic.Both inductive and deductive method of inquiry was adopted, starting with qualitative grounded theory as antecedent variables were bushed in linguistic form. The developed conceptual model, which was demonstrated by 28 items, measuring 6 constructs which tested the behavioral side of the ethical practices, in quantitative phase. The proposed "Ethical work behavior model" was studied for generalizability by smart-PLS and test for [a] the loadings of studied items on their anticipated latent variables and also test [b] the proposed relationship among a set of dependent and independent constructs. Result of 168 distributed questioners in 30 different academic base organizations, indicates that both situational and individual leverage can explain and predict the ethical work behavior.
\end{abstract}

Keywords: Ethical Work Behavior; Organizational Code of Ethic; Mixed Method; Ethical Work Behavior Model

\section{Introduction}

The code of ethics was on the top concern of both business and society. This scenario has made Heyler et al. [1] to assert the importance of the ethical issues in today's world activities. Studding business disasters revealed the ethical dereliction as the main source of debates about the business success or failure [1]. Crane $\&$ Matten [2] have equally argued that, the issues of ethics should not be underrated if business organization planning to succeed and give value to their stakeholders. And therefore, business executives are required to maintain the ethical qualities in their selfconcept and behavior while they are involving in issues such as leadership, business responsibilities for corporate layoffs, closures, and downsizing.

Previous literature mostly focused on relationship between dimension of ethics and the organizational performance; however, the cognitive influence of the code of ethics which deals with the real practice, such as ethical leadership has been left uncovered [3].

A few theories explained how ethical issues should be related in organizational practices. Previous approaches in study of decision making related to the organizational code of ethics typically emphasized either the role of individual characteristics or the role of situational features in behavior which are not bounded to ethical concern; however, it believes, the most important influences existed between individual and situational variables. Therefore, the gap considered by this research is the significance of the code of ethics addressed by individual's cognitive response $[4,5]$.

This issue was associated with using both inductive and deductive approaches and proposed the "ethical behavior model" that gives credence to organizational ethical behavior through integration role of situational and individual cognitive response.

\section{Ethical Decision-Making}

What people ought to do, usually in terms of specific quality of behavior, accepted norm, responsibilities, and benefits to society are well defined as code of ethics [1]. Ethical decision-making is part of cognitive components of response regarding some aspect considered as illegal or factors violates social norms. The emotional components of the ethical behavior include feelings, moods, sentiments, and attitudes toward an issue or decision [6], whereas the cognitive components include conscious beliefs, knowledge, perceptions, and judgments [7].

Many theories have been developed such as ethical-decision making theory in marketing which introduced by Dubinsky \& Loken [8]; four-components model by Rest [9]; contingency theory by Ferrell \& Gresham [10]; general theory of marketing ethics by Hunt \& Vitell [11]; person-situation interactionism model by Treviño [12] and issue contingent model by [13].

While, yet the impact of individual and situational variables on human behavior has been examined in depth. The previous models sustain the influence of emotional component of individual judgments and behaviors, as they placed more concern on the effect of a person feelings, religiosity or convergence with law. Although, ethics are often equated with feelings, nevertheless, acting ethically is bounded on feelings alone, as feelings may lead one individual to do what is right but lead the other person in different way. On the other hand, while, most religions advocate high ethical standards, nevertheless, ethics should not be identified with religion, as some individuals do not stand their ethics solely upon a particular religious teaching. Moreover, acting ethically is not the same as acting according to the law; although many laws incorpo- 
rate with ethical standards to which most citizens subscribe, nevertheless, it can deviate from that as well.

\section{Research Objectives}

This research attempted to understand deep into the constructs of the code of ethics. The objective therefore is, to unpack different dimensions of the cognitive response and clarifies different antecedent's instruments of the code of ethics.

\section{Methodology}

In attempt for a rigorous study about the causes and effects of code of ethics, the methodology which has been used in this research was grounded based on post-positivism philosophy using both inductive and deductive method of data inquiry. The first phase was based on grounded theory of Strauss and Corbin's approach, while the second phase was designed for generalizing the proposed model by logical regression concordance of multiple premises. This attempt was based on calling for further mixedmethods research by $[1,14]$

Qualitative exploration was an essential instrument of this research as the saturated level of data brought new insights among different constructs [15]. The proposed model therefore, draws attention to the growing need of more understanding about naturalistic settings, the importance of cognitive context, and the complexity of implementing social change.

Quantitative was has been used to test the model by using a survey instrument which was partly adapted from previous studies and manipulated to be suitable for the research constructs, while its validity was systematically ensured.

\section{Instrumentation}

The questioner has been developed following the recommended procedures of [16]. Conceptual validation was done through sorting procedure as proposed by [17]. The research instrument includes a total number of 28 items, measuring 6 constructs which are shown in figure 2. Seven-point Likert scales used to measure the items which were adapted and adopted from previous validated researches.

The validation process was done through two phases. First, the questioner was validated by 4 academicians and 4 experts in similar topic, in order to insure the clarity and correctness of the items. Second, two rounds sorting procedure has been applied based on [17]. The internal reliability was checked based on Kappa statistic [18] and items placement ratio also applied based on [17]. Values have been calculated, and the results of both rounds were greater than 0.89 which is greater than 0.65 . Therefore, based on Kappa scores, it is confirmed that the scales are highly reliable and considered to be acceptable [17].

\section{Data Collection}

The main source of data in qualitative phase was collected through semi-structured interviews of top executives from three ranked academic institutes. In all the cases, the respondents were allowed to state their stories as they see themselves involved in real life situations by using of open-ended questions. However, to have effective interview sessions, an interview protocol was followed until the saturation level.

Considered organizations were all involved in education and training as the unit of analyses. Total 10 expert participants are key top principal who represent the organizational strategic decision makers; which their detailed information kept confidential due to sensitivity of the issue related the code of ethic. Should be note, the comparison between interviews were done in order to ensure about the consistency of the received information. To ensure accuracy, during the interviews, tape-recorders were used and tran- scription was eventually sent to the interviewees to double check the precision of collected information.

In quantitate phase, the questioner has been distributed among the head of departments as organizations' decision makers in 30 different educational institutes, operating in capital city of Iran [Tehran], including schools, institutes, universities and training centers. Total numbers of 168 questioners have been distributed, while only 151 have been returned; nevertheless, only 143 were completed and able to be used for further processes. Collected data analyzed by using the ver 2 of Smart-PLS software.

\section{Qualitative Phase - Grounded Theory Method}

Replication logic developed by Glaser [19] was followed for the experts' selection, as this is important for cases that are multiple in natures [20]. Furthermore, the researchers also compared data and theory as analysis began with collection of data while emphasis was laid on both emergence of theory with evidence and an incremental approach [19]. For analyzing each case, research problems were formulated at the beginning, where some potentially significant variables from existing literature and grounded theory were analyzed [21].

There were several in-depth sessions of interviews based on grounded theory design, while a within-case analysis was also conducted after the participant's histories were conducted. Founded pattern which is unique within the finding, pursued for generalization across cases [22]. Results are briefly explained in next.

\subsection{Ethical Work Behavior Model}

It was found after cross cases analysis of interviews, that the ethical work behavior can be explained by a combination of individual and situational leverage. While factors such as administrative role and the longer working experience, the larger size of the organization were possessing productive catalytic mechanism; nevertheless, some degree of code of ethics such as antecedents believes found more effective in this construct. Especially leaders are tend to build relationships with their affiliates and make them feel working in more democratic environment and increase the perception about their higher degree of ethical leadership. As illustrated in the proposed ethical work behavior model, [Figure 1] the theory of planned behavior can be seen as a specific application in the workplace.

\subsection{Organizational Climate as the Situational Leverage}

The organizational climate reflects the collective views of working practices and the different culture in which organizational units or members have conducted organizational operations [23]. As such, organizational climate often reflects the values, norms, and traditions that have developed over time and influence current employee processes.

\subsection{Behavioral Belief}

According to Ajzen's theory of planned behavior [24], behavioral belief represents in form of expected outcome in the proposed model. Opportunities reflect the ethical leadership within an organization who set many of the policies and rules that directly influence an individual's opportunity to behave ethically. The components of expected outcome include integrity, established reinforcements, communication, and process orientation.

\subsection{Normative Beliefs}

According to Ajzen's theory of planned behavior [24], normative beliefs pertain to the approval or disapproval that others are expected to express after performing a behavior. This study classified normative beliefs according to two dimensions of social 
norms and organizational rules and policies. Should be noted that, organizational rules and policies reflect the organizational climate and the norms and rules that stimulated from the organizational climate. The connection between behavioral belief and normative beliefs is because of being influenced and influencing role of leaders directly and indirectly on subordinates' behavior. It means that, these ethical leaders can ultimately effect on ethical work behavior or decrease unethical work behavior via social influence including "personal interactions and set expectations and the promotion of such behavior to others through communication, reinforcement, and decision making.

\subsection{Intention}

Intention is considered as immediate antecedent of one's behavior and defined as an indication of a person's readiness to perform an assumed behavior. Specifically, research on intention-behavior relationship has illustrated that the organizational climate can predict ethical work behavior. Intention stems from the negative evaluation of others and a belief in being ethically superior to them. In this study we argue, intention while does not have solid change in behavior nevertheless, it causes self-cognitive changes. Therefore, it was proposed that intention toward a behavior is positively related to ethical work behavior

\subsection{Individual Moderators}

Social constructionists posit that cognitive beliefs congruence influence ethical behaviors not through reflective processes but rather the perception of socially learned responses to emotional arousal [6]. This study classified cognitive beliefs according to two dimensions ethical identity and active emotions. This finding may indicate that individuals experiencing self-conscious emotions do not engage in active/direct unethical behaviors but rather more passive-aggressive behaviors. Ethical identity is defined as routine ethical traits in ones' self-concept and behavior. Trait aspects of ethical identity include compassion, trust, and caring [7], whereas social aspects of ethical identity are based on the individual's self-concept of the groups and networks of which he or she feels a part [e.g., religious or political groups].

In despite of the existence of ethical policies and emphasis on organizational procedures, nevertheless, because of the impact of individual's emotions, a workplace may still be going by unethical work behaviors. Even with all the rational areas accounted for human behavior is influenced by one's cognitive beliefs congruence. Modern cognitive psychologists often consider actives emotions to be the antagonists of rationality. While according to participant's expressions in this research we argue that one would make better decisions with the influence of emotions, and in fact the human brain cannot make sound decisions without active emotions and logic which emotions must interact for it.

\subsection{Ethical Work Behavior}

This study utilized the relatively broad definition of unethical work behaviors as a variety of harmful behaviors that individuals perform and negatively impact to their peers, work teams, and the organization [e.g., gossip, bullying, deliberate exclusion, hostility] which are subjected to be judged according to generally accepted ethical norms or behavior. The different terminology used by participants referring to unethical work behavior, includes organizational misbehavior, counterproductive work behaviors, and antisocial behavior. For example, participant P.C.1, clearly defined the unethical work behavior as "violation of organizational norms and rules". Workplace bullying involves a pattern of negative acts characterized by the features of isolation, humiliation, and intimidation. The persistence of a negative behavior is particularly important to the study because it moves the focus from negative acts to negative relationships and distinguishes workplace unethical behavior from other forms of ethical behavior. Previous research on human aggression, reports that the persistence of a negative behavior in the workplace, which can decrease productivity and downsize the job satisfaction [23], involves destructive attitudes such as negativity, bitterness, procrastination, "forgetting" to do something, and intentional inefficiency. Passive aggressive behaviors include spreading rumors, gossiping, attempting to turn others against the target, deliberately excluding the target, and withholding information [25]. Passive aggressive behaviors cost the organization time, energy, and money [25].

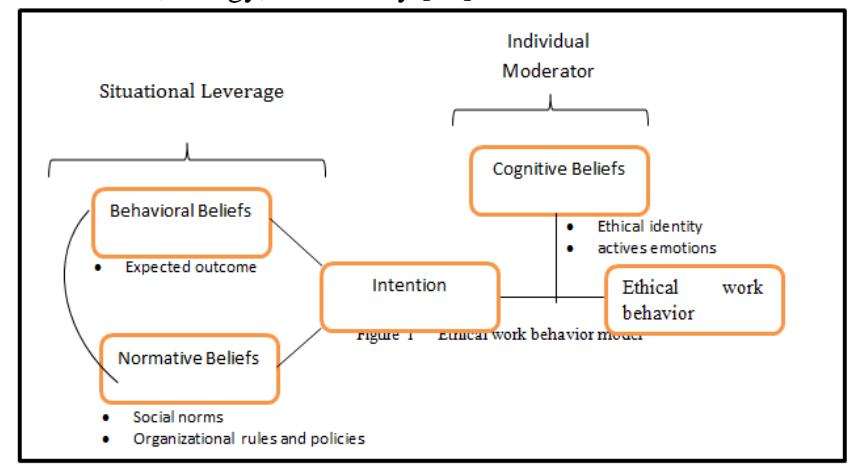

Fig. 1: Proposed Model "Ethical Work Behavior Model"

\section{Quantitative Phase - Statistical Analysis}

Two types of test have been conducted, first test the loadings of observed items on their expected latent variables which is called the measurement model test, second the structural model test which is concentrated on the proposed relationship among a set of dependent and independent constructs [26].

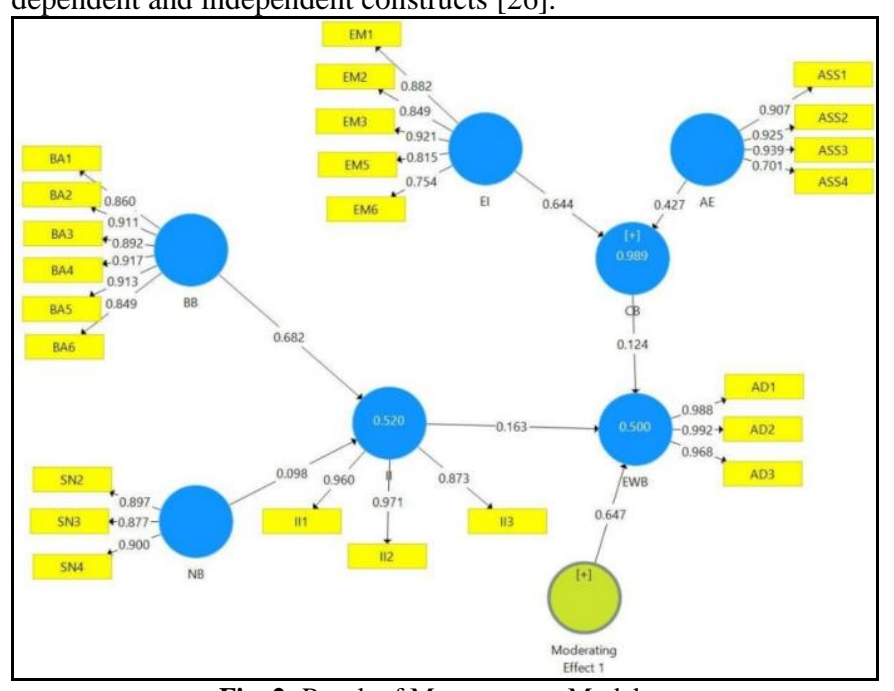

Fig. 2:.Result of Measurement Model

For this research, all the constructs are reflective; therefore, the Cronbach's alpha reliability coefficient can be used to measure the accuracy of measuring instrument [27][Cronbach, 1951]. Score of 0.50 to 0.60 would be sufficient [28] and the score recommended is 0.70 [17]. In the Analyzing we found that all the constructs got a Cronbach's alpha value higher than the recommended value 0.70 . The Convergent validity refers to the degree of the relation between two measures of constructs that theoretically should be related, is calculated using three tests: [1] item reliability which means the loading of each item has to be more than 0.707 [29]. [2] Composite reliability which should be greater than the recommended 0.7 [30] and [3] average variance extracted [AVE] are greater than the required 0.50 of all the indicators [30]. For our test, the results show that all our constructs show satisfactory levels of convergent validity.

The factors clarified $50 \%$ of total cumulative variance. The Factor loading values are higher than the generally accepted number of 0.5 of all items on their intended constructs [31]. All factors have an eigenvalue greater than one. This shows the constantly of the factors which means that the items presenting the factor well [32]. 
The correlations between the measures of two constructs were examined for the second test of discriminant validity. The Results show that more variance is shared between the construct and its indicators than with another construct representing a different set of indicators [29]. This indicates adequate discriminant validity.

Thus, results show that all reflective constructs exhibit adequate levels of internal consistency, convergent validity and discriminant validity.

For the structural model testing, we used the [R2] and path coefficients. R2 of $10 \%$ indicates a substantive explanatory power [33] This is shown in the proposed model as higher than $50 \%$. Also the moderating effect of the cognitive beliefs is clearly shown. [Figure 2] Thus, we contemplate that our proposed model holds an acceptable level of predictive validity. Our model fulfills the criteria of a good model fit, as obvious from the results of our measurement model and structural model evaluation.

\section{Discussions and Implications}

Most previous researches have focused on assessing the ethics of final decisions without attempting to investigate the processes underlying ethical decision-making. This research tried to explore and examine the antecedents [i.e. organizational climate and leader's characteristics] and also consequences factors [organizational code of ethics]. Conducted in-depth inductive study, validate the proposed framework and relative propositions. Based on the results, the ethical work behavior can cultivate by two groups of influential factors of situational leverage and individual moderator. It is found that, while individuals are highly influenced by their superiors and peers in daily processes, including work interactions: nevertheless, these individuals come to social situations with their own unique set of experiences that have molded the way that they see rules and norms. Such their behavioral responses are a function of mental processes that begin with active emotion and expected ethical identity. Basically, the main theme of this study has implications for self-conscious and active emotions relate to employee work behavior.

In similar with Shapiro \& Stefkovich [3] of concept of moral intensity, this research also found that individuals may consider the probability of consequences before engaging in a particular behavior. In the case of the emotion of guilt, as employees experiencing fault may feel that they have ruined their reputation, and making others a negative wave for further destroying of their reputations. These results illustrate a relationship between unethical work behavior and specific emotions [i.e., guilt, shame, contempt] and extending the current literature on emotions and unethical work behaviors.

Furthermore, it was discovered that those with strong ethical identity will develop a positive capacity to act ethically, accept ethical goals, and direct efforts to deal with ethical challenges in a manner consistent with their ethical identity. Such individuals choose to behave in accordance with their true self-perception or code of ethic that is matched to their ideal ethical concept. It can be interpreted that individuals who have a high level of ethical identity display significantly lower levels of passive-aggressive behaviors compared to those with a low level of ethical identity. In other word individuals who decide not to engage in more covert, passive aggressive behaviors may require a stronger ethical identity. It is found that, importance of ethical identity and the unique properties that effect on this individual characteristic.

Regarding the strong ethical organizational climate characterized by common norms that motivate managers and employees for integrity and value the code of ethic instead of fear of consequences. This finding may be attributed to the types of unethical behaviors and explain the existence of a significant relationship between organizational climate and ethical leadership; specifically, that ethical leaders directly influences organizational norms and expectations regarding unethical work behavior.

This finding indicates the need of organizations and supervisors to work with employees to identify healthy coping and regulation techniques to provide them with more ethical ways and release their emotional burdens, existing conflict through increased communication and conflict management skill. Organizations that fail to establish structure of communicating ethical standard across organization may experience more unethical work behaviors than organizations that have clearly articulated ethical standards and the consequences of deviating. Organization therefore should at tempt to assess the impact of influential emotions based on the understanding that accurate identification of how and when emotions interact to affect decisions and behaviors.

In conclusion, it is arguing that active emotion and ethical identity moderated the ethical work behavior as self-cognitive beliefs congruence influence self-knowledge and normative expectation. Based on this argument, the previous attempts that measures ethical work behavior without considering active emotions and ethical identity may not accurately capture one's certain characteristically ethical traits in ones' self-concept and behavior and basically the future researcher should be consider the role of self-cognitive beliefs in either form of active emotions and ethical identity, as this moderator yield more insight into executives' motivation behind their ethical work behavior including behavioral beliefs, social norms, or belief in a higher being.

\section{Directions for Future Research}

This study provides preliminary insight into the effects of emotions, as well as the effects of their interaction with other situational and individual factors, on ethical decision-making and unethical work behaviors that will guide future research. Nevertheless, the question remains to be answered of how these constructs changes and evolves over time? Future research, may argue on how ethical leadership and the cognitive mechanism may negatively relate to unethical work behavior and produce different effect on the organizational administrative. This research laid emphasis on replication logic developed by Yin [20] and Strauss and Corbin [15] with a focus on the theoretical replication; and future researchers should make use of social learning theory, social exchange theory and social information processing theory. This research studied knowledge related institutions; therefore, care and caution must be taken in the application of our model to other market or industries. However, the finding can have substantial contribution to both managerial ethic and administrative theories.

\section{References}

[1] Heyler SG, Armenakis AA, Walker AG, Collier DY. A qualitative study investigating the ethical decision making process: A proposed model. The Leadership Quarterly. 2016;27[5]:788-801.

[2] Crane A, Matten D. Business ethics: Managing corporate citizenship and sustainability in the age of globalization: Oxford University Press; 2016.

[3] Shapiro JP, Stefkovich JA. Ethical leadership and decision making in education: Applying theoretical perspectives to complex dilemmas: Routledge; 2016.

[4] Sami A, Jusoh A, Qureshi MI. Does Ethical Leadership Create Public Value? Empirical Evidences from Banking Sector of Pakistan. International Review of Management and Marketing. 2016;6[4S].

[5] Sami A, Jusoh A, Mahfar M, Qureshi MI, Khan MM. Role of Ethical Culture in Creating Public Value. International Review of Management and Marketing. 2016;6[4S].

[6] Babalola MT, Stouten J, Camps J, Euwema M. When do ethical leaders become less effective? The moderating role of perceived leader ethical conviction on employee discretionary reactions to ethical leadership. Journal of Business Ethics. 2017:1-18.

[7] Hinojosa AS, Gardner WL, Walker HJ, Cogliser C, Gullifor D. A review of cognitive dissonance theory in management research: Opportunities for further development. Journal of Management. 2017;43[1]:170-99.

[8] Dubinsky AJ, Loken B. Analyzing ethical decision making in marketing. journal of Business Research. 1989;19[2]:83-107.

[9] Rest JR. Moral development: Advances in research and theory. 1986. 
[10]Ferrell OC, Gresham LG. A contingency framework for understanding ethical decision making in marketing. The Journal of Marketing. 1985:87-96.

[11]Hunt SD, Vitell S. A general theory of marketing ethics. Journal of macromarketing. 1986;6[1]:5-16.

[12] Trevino LK. Ethical decision making in organizations: A personsituation interactionist model. Academy of management Review. 1986;11[3]:601-17.

[13] Jones TM. Ethical decision making by individuals in organizations: An issue-contingent model. Academy of management review. $1991 ; 16[2]: 366-95$.

[14] Todd EM, Torrence BS, Watts LL, Mulhearn TJ, Connelly S, Mumford MD. Effective practices in the delivery of research ethics education: A qualitative review of instructional methods. Accountability in research. 2017;24[5]:297-321.

[15] Strauss A, Corbin J. Basics of qualitative research: Procedures and techniques for developing grounded theory. Thousand Oaks, CA: Sage; 1998.

[16] Churchill Jr GA. A paradigm for developing better measures of marketing constructs. Journal of marketing research. 1979:64-73.

[17] Moore GC, Benbasat I. Development of an instrument to measure the perceptions of adopting an information technology innovation. Information systems research. 1991;2[3]:192-222.

[18] Cohen J. A coefficient of agreement for nominal scales. Educational and psychological measurement. 1960;20[1]:37-46.

[19] Glaser B. Discovery of grounded theory: Strategies for qualitative research: Routledge; 2017.

[20] Yin RK. Qualitative research from start to finish: Guilford Publications; 2015

[21] Merriam SB, Tisdell EJ. Qualitative research: A guide to design and implementation: John Wiley \& Sons; 2015

[22] Yazan B. Three approaches to case study methods in education: Yin, Merriam, and Stake. The Qualitative Report. 2015;20[2]:134-52.

[23] Ching SL, Kee D, Tan CL. The impact of ethical work climate on the intention to quit of employees in private higher educational institutions. Journal of southeast Asian research. 2016:1-11.

[24]Ajzen I. The Theory of Planned Behavior. Organizational Behavior and Decision Processes. Massachusetts. Academic Press. Inc; 1991.

[25]Dang CT, Umphress EE, Mitchell MS. Leader social accounts of subordinates' unethical behavior: Examining observer reactions to leader social accounts with moral disengagement language. Journal of Applied Psychology. 2017;102[10]:1448.

[26] Gefen D, Straub D, Boudreau M-C. Structural equation modeling and regression: Guidelines for research practice. Communications of the association for information systems. 2000;4[1]:7.

[27] Cronbach LJ. Coefficient alpha and the internal structure of tests. psychometrika. 1951;16[3]:297-334.

[28] Nunnally Jr JC. Introduction to psychological measurement. 1970.

[29] Chin WW. The partial least squares approach to structural equation modeling. Modern methods for business research. 1998;295[2]:295336

[30] Fornell C, Larcker DF. Evaluating structural equation models with unobservable variables and measurement error. Journal of Marketing Research. 1981:39-50

[31]Hair JF, Black WC, Babin BJ, Anderson RE, Tatham RL. Multivariate data analysis: Prentice hall Upper Saddle River, NJ 1998.

[32] Johnson DE. Applied multivariate methods for data analysts: Duxbury Resource Center; 1998.

[33]Falk R, Miller N. A primer for soft modeling University of Akron Press Akron. OH; 1992. 